\title{
EVALUATION OF COFFEE GROUND AS A FEEDSTUFF IN PRACTICAL DIETS FOR OLIVE FLOUNDER PARALICHTHYS OLIVACEUS
}

\author{
Samad Rahimnejad, Sang-Min Lee* \\ Department of Marine Bioscience and Technology, Gangneung-Wonju National University, \\ Gangneung 25457, Korea
}

\begin{abstract}
A 10-week feeding trial was carried out to investigate the feasibility of using spent coffee ground (CG) as a potential feed ingredient for olive flounder. Growth, feed utilization, body composition and antioxidant enzyme activity were examined. A control diet was formulated and three other diets were prepared to contain 5, 10 or $15 \%$ CG (designated as Con, CG5, CG10 and CG15, respectively) by replacing for wheat flour. At the end of the feeding trial, fish fed the CG5 diet exhibited significantly $(P<0.05)$ higher growth performance than those fed the control diet. Also, fish fed the CG10 diet had a comparable growth to that of the control group, but further increase of dietary CG inclusion level to $15 \%$ resulted in significant decrease of growth performance. Fish fed the CG15 diet showed significantly lower feed efficiency and protein efficiency ratio than other treatments. Significantly lower muscle protein content was observed in fish fed CG15 diet compared to the control. Significant reduction in plasma cholesterol concentration was found in fish fed CG15 diet compared to control. No significant changes were found in alkyle and superoxide radicals scavenging activities of plasma, muscle and liver among dietary treatments. Also, liver total protein, total antioxidant capacity, catalase and glutathione peroxidase activities were not significantly influenced by dietary inclusion of CG. According to these results, we concluded that CG can be included up to $10 \%$ in the diet for olive flounder without any adverse effects on growth, feed utilization and antioxidant enzyme activity.
\end{abstract}

\section{KEY WORDS}

Olive flounder, Coffee ground, growth performance, feed utilization, antioxidant enzyme activity

${ }^{*}$ Corresponding author: Tel.: +82 33640 2414; Fax: +82 336402955.

E-mail address: smlee@gwnu.ac.kr 